\title{
NOTE ON THE SMALLEST ROOT OF THE INDEPENDENCE POLYNOMIAL
}

\author{
PÉTER CSIKVÁRI
}

\begin{abstract}
One can define the independence polynomial of a graph $G$ as follows. Let $i_{k}(G)$ denote the number of independent sets of size $k$ of $G$, where $i_{0}(G)=1$. Then the independence polynomial of $G$ is$$
I(G, x)=\sum_{k=0}^{n}(-1)^{k} i_{k}(G) x^{k} .
$$

In this paper we give a new proof for the fact that the root of $I(G, x)$ having the smallest modulus is unique and it is real.
\end{abstract}

\section{INTRODUCTION}

Throughout the paper $G$ is a graph without multiple edges and loops. The independence polynomial of the graph $G$ is defined as follows. Let $i_{k}(G)$ denote the number of independent sets of size $k$ of $G$, where $i_{0}(G)=1$. Then the independence polynomial of $G$ is

$$
I(G, x)=\sum_{k=0}^{n}(-1)^{k} i_{k}(G) x^{k} .
$$

We note that in general, $I(G,-x)$ is called the independence polynomial in the literature. We will see that it is much more convenient to work with this form. Since the connection between the two forms is very simple, it will not cause any confusion. We also mention that several authors consider the clique polynomial of the graph $G$, which is $I(\bar{G}, x)$ in our notation. Surprisingly, slightly more convenient to work with the independence polynomial than with the clique polynomial.

Let $\beta(G)$ be the smallest real root of the independence polynomial. It is known that it exists and it is in the interval $(0,1]$ (see [5]). It is also well-known that if $\rho$ is another root of the independence polynomial, then $|\rho| \geq \beta(G)$ [5]. It was only proved much later by Goldwurm and Santini [7] that in fact, the stronger statement $|\rho|>\beta(G)$ holds too. On the other hand, their proof heavily relies on the theory of formal languages. However it was not the aim of Goldwurm and Santini, their theorem had an important application, namely, it filled a gap in a proof of Fisher [3] on the minimal

2000 Mathematics Subject Classification. Primary: 05C31.

Key words and phrases. independence polynomial, root.

The author is partially supported by the Hungarian National Foundation for Scientific Research (OTKA), grant no. K81310 and by grant no. CNK 77780 from the National Development Agency of Hungary, based on a source from the Research and Technology Innovation Fund. The author is also partially supported by MTA Rényi "Lendület" Groups and Graphs Research Group. 
number of triangles of a graph with prescribed number of edges, see Remark 3.3 .

In this short note we give a considerably simpler proof for this theorem.

Theorem 1.1. Let $\rho$ be a root of the independence polynomial $I(G, z)$ different from $\beta(G)$ then $|\rho|>\beta(G)$.

The paper is organized as follows. We end this Introduction by presenting our notations. In Section 2 we collect the required tools from complex function theory. In Section 3 we prove Theorem 1.1. We end the paper with some concluding remarks on the weighted version of Theorem 1.1.

Notation 1.2. Throughout the paper we will consider only simple graphs. As usual $G=(V, E)$ will denote a graph with vertex set $V(G)$ and edge set $E(G)$. Let $e(G)$ be the number of edges, i.e., $|E(G)|=e(G)$. The set of neighbors of the vertex $v$ will be denoted by $N_{G}(v)$; if there is no confusion we will simply write $N(v)$ instead of $N_{G}(v)$. The closed neighborhood of the vertex $v$ is $N_{G}[v]=N_{G}(v) \cup\{v\}$. The degree of the vertex $v$ will be denoted by $d(v)=\left|N_{G}(v)\right|$.

For $S \subset V(G)$ the graph $G-S$ denotes the subgraph of $G$ induced by the vertices $V(G) \backslash S$. If $e \in E(G)$ then $G-e$ denotes the graph with vertex set $V(G)$ and edge set $E(G) \backslash\{e\}$.

\section{LEMMAS FROM COMPLEX ANALYSIS}

In this part we collect the required background from complex analysis. We use only two theorems from complex analysis, both theorems are well-known and they can be found in [6].

Lemma 2.1 (Pringsheim's theorem). [6] If $f(z)$ is representable at the origin by a series expansion that has non-negative coefficients and radius of convergence $R$, then the point $z=R$ is a singularity of $f(z)$.

Let

$$
f(z)=\sum_{n=0}^{\infty} f_{n} z^{n}
$$

be analytic in $|z|<R$, and assume that $f_{n} \geq 0$ for $n \geq 0$. Then for any $|s|<R$ we have

$$
|f(s)|=\left|\sum_{n=0}^{\infty} f_{n} s^{n}\right| \leq \sum_{n=0}^{\infty}\left|f_{n}\right||s|^{n}=\sum_{n=0}^{\infty} f_{n}|s|^{n}=f(|s|) .
$$

By investigating the case of equality we obtain Lemma 2.3. To prepare it, we need some terminology.

Definition 2.2. Let

$$
f(z)=\sum_{n=0}^{\infty} f_{n} z^{n}
$$


NOTE ON THE SMALLEST ROOT OF THE INDEPENDENCE POLYNOMIAL 3

Let $\operatorname{Supp}(f)=\left\{k \mid f_{k} \neq 0\right\}$. The sequence $\left(f_{n}\right)$ as well as $f(z)$ admits a span $d$ if for some $r$, there holds

$$
\operatorname{Supp}(f) \subseteq r+d \mathbb{Z}_{\geq 0}=\{r, r+d, r+2 d, \ldots\} .
$$

The largest span, $p$, is the period, all other spans being divisors of $p$. If the period is equal to 1 , then the sequence $\left(f_{n}\right)$ and $f(z)$ are said to be aperiodic.

Lemma 2.3. [6] Let $f(z)$ be analytic in $|z|<R$ and have non-negative coefficients at 0 . Assume that $f$ does not reduce to a monomial and that for some non-zero $s$ with $|s|<R$, one has

$$
|f(s)|=f(|s|) .
$$

Then the following holds, (i) the argument of $s$ must be commensurate to $2 \pi$, i.e., $s=|s| e^{i \theta}$ with $\theta / 2 \pi=\frac{r}{p} \in \mathbb{Q}$ (an irreducible fraction) and $0<r<p$ : (ii) $f$ admits $p$ as a span.

In fact, we will only need that if the sequence $\left(f_{n}\right)$ is aperiodic, then $|f(s)| \neq f(|s|)$ if $s$ is non-zero, non-positive.

\section{Generator Functions of the independence polynomial}

Throughout this section we will use the following simple recursive formulas for the the independence polynomial.

Lemma 3.1. [9] (a) If $G$ is non-connected graph with connected components $H_{1}, \ldots, H_{k}$ then

$$
I(G, z)=\prod_{j=1}^{k} I\left(H_{j}, z\right) .
$$

(b) Let $v$ be an arbitrary vertex of the graph $G$. Then

$$
I(G, z)=I(G-v, z)-z I(G-N[v], z) .
$$

(c) If $e=(u, v) \in E(G)$, then we have

$$
I(G, z)=I(G-e, z)-z^{2} I(G-N[u]-N[v], z) .
$$

The next lemma is the main tool to prove Theorem 1.1.

Lemma 3.2. Let $G$ and $H$ be graphs and set

$$
\frac{I(H, z)}{I(G, z)}=\sum_{k=0}^{\infty} r_{k}(H, G) z^{k} .
$$

Then

(a) If $H$ is a proper induced subgraph of $G$, then $r_{k}(H, G)>0$ for $k \geq 0$.

(b) If $H$ is a proper subgraph of $G$, then $r_{k}(H, G)>0$ for $k \geq 2$.

Proof. (a) We prove the statement by induction on the number of vertices of $G$. If $|V(G)|=1$ then $|V(H)|=0$ and

$$
\frac{I(H, z)}{I(G, z)}=\frac{1}{1-z}=\sum_{j=0}^{\infty} z^{j} .
$$


It is enough to prove the statement for $H=G-u$, where $u$ is an arbitrary vertex of $G$. Indeed, if $H=G-S$ for some $S=\left\{u_{1}, \ldots, u_{k}\right\} \subseteq V(G)$, then

$$
\frac{I(H, z)}{I(G, z)}=\frac{I\left(G-u_{1}, z\right)}{I(G, z)} \frac{I\left(G-\left\{u_{1}, u_{2}\right\}, z\right)}{I\left(G-u_{1}, z\right)} \cdots \frac{I\left(G-\left\{u_{1}, \ldots u_{k}\right\}, z\right)}{I\left(G-\left\{u_{1}, \ldots, u_{k-1}\right\}, z\right)} .
$$

By induction we already know that all terms except the first one have a power series expansion of positive coefficients. Hence it is enough to prove that $r_{k}(G-u, G)>0$ for all $k \geq 0$.

Now we use the recursive formula

$$
I(G, z)=I(G-u, z)-z I(G-N[u], z) .
$$

We have

$$
\begin{aligned}
& \frac{I(G-u, z)}{I(G, z)}=\frac{I(G-u, z)}{I(G-u, z)-z I(G-N[u], z)}= \\
& =\frac{1}{1-z \frac{I(G-N[u], z)}{I(G-u, z)}}=\sum_{k=0}^{\infty}\left(z \frac{I(G-N[u], z)}{I(G-u, z)}\right)^{k} .
\end{aligned}
$$

If $G-N[u]$ is a proper subgraph of $G-u$ then by induction all the coefficients of the power series $\frac{I(G-N[u], z)}{I(G-u, z)}$ are positive and so all the coefficients of $\frac{I(G-u, z)}{I(G, z)}$ are positive. If $G-N[u]=G-u$ then $\frac{I(G-u, z)}{I(G, z)}=\frac{1}{1-z}$ and we are done again.

(b) We prove the claim by induction on the number of edges of $G$. If $e(G)=0$ and $|V(G)|=n,|V(H)|=k$, where $k<n$ then

$$
\frac{I(H, z)}{I(G, z)}=\frac{(1-z)^{k}}{(1-z)^{n}}=\sum_{k=0}^{\infty}\left(\begin{array}{c}
n-k-1+j \\
n-k-1
\end{array}\right) z^{j}
$$

Thus the statement is true in this case. Just like in the case of the proof of part $(a)$ it is enough to prove that $r_{k}(G-e, G)>0$ for all $k \geq 2$. Indeed, if $E(H)=E(G)-\left\{e_{1}, \ldots, e_{k}\right\}$ and $|V(G)|-|V(H)|=s$ then

$\frac{I(H, z)}{I(G, z)}=\frac{I\left(G-e_{1}, z\right)}{I(G, z)} \frac{I\left(G-\left\{e_{1}, e_{2}\right\}, z\right)}{I\left(G-e_{1}, z\right)} \ldots \frac{I\left(G-\left\{e_{1}, \ldots e_{k}\right\}, z\right)}{I\left(G-\left\{e_{1}, \ldots, e_{k-1}\right\}, z\right)} \frac{1}{(1-z)^{s}}$.

By induction we already know that all terms except the first one have a power series expansion of the required form. Hence it is enough to prove that $r_{k}(G-e, G)>0$ for all $k \geq 2$. Now we use the second recursive formula. If $e=(u, v) \in E(G)$, then we have

$$
I(G, z)=I(G-e, z)-z^{2} I(G-N[u]-N[v], z) .
$$

We have

$$
\begin{gathered}
\frac{I(G-e, z)}{I(G, z)}=\frac{I(G-e, z)}{I(G-e, z)-z^{2} I(G-N[u]-N[v], z)}= \\
=\frac{1}{1-z^{2} \frac{I(G-N[u]-N[v], z)}{I(G-e, z)}}=\sum_{k=0}^{\infty}\left(z^{2} \frac{I(G-N[u]-N[v], z)}{I(G-e, z)}\right)^{k} .
\end{gathered}
$$

Since $G-N[u]-N[v]$ is a proper induced subgraph of $G-e$, we have by part (a) that all the coefficients of the power series $\frac{I(G-N[u]-N[v], z)}{I(G-e, z)}$ are positive and $r_{k}(G-e, G) \geq 2$ for $k \geq 2$. 
Remark 3.3. From a theorem of Cartier and Foata [1] one can deduce that $r_{k}(\emptyset, G)$ counts the number of words of length $k$ in a certain 'semi-Abelian' monoid. This deduction is explained in [2], where Fisher also gave a direct proof of this combinatorial meaning. So it was known that $r_{k}(\emptyset, G) \geq 0$. Probably, a similar interpretation of $r_{k}(H, G)$ can be given too.

We mention that Fisher [3] used the connection between the independence polynomial and this counting problem to give a sharp lower bound for the number of triangles in a graph $G$ with $n$ vertices and $e$ edges if $n^{2} / 4 \leq e \leq$ $n^{2} / 3$. His proof contained a small gap: he used $|\rho|>\beta(G)$ for $\rho \neq \beta(G)$, but he only proved $|\rho| \geq \beta(G)$. We also note that the problem of giving (asymptoticaly) sharp lower bounds for the number of cliques of size $k$ in a graph with fix number of vertices and edges was resolved by Razborov [11] $(k=3), \mathrm{V}$. Nikiforov [10] $(k=3,4)$ and C. Reiher [12] $(k \geq 3)$. They used completely different machinery than Fisher.

Corollary 3.4. $[4,5]$ Let $\beta(G)$ be the convergence radius of $\frac{1}{I(G, z)}$. Then $\beta(G)$ is a root of the independence polynomial $I(G, z)$, and it has the smallest modulus among the roots of $I(G, z)$. Let $H$ be a subgraph of $G$. Then $\beta(G) \leq$ $\beta(H)$.

Proof. Let

$$
\frac{1}{I(G, z)}=\sum_{k=0}^{\infty} r_{k}(G) z^{k}
$$

Clearly, $r_{k}(G)=r_{k}(\emptyset, G)>0$ for all $k \geq 1$. Hence Pringsheim's theorem (Lemma 2.1) already gives the first part of the claim.

Since

$$
\frac{1}{I(G, z)}=\frac{I(H, z)}{I(G, z)} \frac{1}{I(H, z)}
$$

we obtain that $r_{k}(G) \geq r_{k}(H)$ if $H$ is a subgraph of $G$. Hence

$$
\beta(G)=\liminf _{k \rightarrow \infty} r_{k}(G)^{-1 / k} \leq \liminf _{k \rightarrow \infty} r_{k}(H)^{-1 / k}=\beta(H) .
$$

Remark 3.5. A very simple proof of Corollary 3.4 can also be found in [8].

At this moment we do not exclude the possibility that some other root of the independence polynomial has modulus $\beta(G)$. We will exclude it later.

Our next aim is to prove that if $H$ is a proper subgraph of the connected graph $G$ then $\beta(G)<\beta(H)$ holds. This is the last step to prove the uniqueness of the $\operatorname{root} \beta(G)$.

Lemma 3.6. Let $G$ be a connected graph and let $H$ be a proper subgraph of $G$. Then $\beta(G)<\beta(H)$ and the multiplicity of the root $\beta(G)$ is 1 .

Proof. We prove the claim by induction on the number of edges of $G$. If $e(G)=0$ or 1 then the claim is trivial. Clearly, it is enough to prove that $\beta(G)<\beta(G-e)$ for any edge $e=(u, v)$. Since $\beta(G) \leq \beta(G-e)$ we only need to prove that $\beta(G) \neq \beta(G-e)$. Assume for a contradiction that $\beta(G)=\beta(G-e)$. Since

$$
I(G, z)=I(G-e, z)-z^{2} I(G-N[u]-N[v], z)
$$


we obtain that $\beta(G)=\beta(G-e)$ is also a root of $I(G-N[u]-N[v], z)$. Hence $\beta(G-N[u]-N[v])=\beta(G-e)$. If $G-e$ is connected, then it is already a contradiction since $G-N[u]-N[v]$ is a proper subgraph of $G-e$ thus $\beta(G-N[u]-N[v])>\beta(G-e)$. If $G-e$ is not connected, then $G-e=H_{1} \cup H_{2}$, where $H_{1}$ and $H_{2}$ are connected graphs. If $u \in V\left(H_{1}\right)$ and $v \in V\left(H_{2}\right)$ then

$$
G-N[u]-N[v]=\left(H_{1}-N[u]\right) \cup\left(H_{2}-N[v]\right) .
$$

Hence

$\beta(G-e)=\min \left(\beta\left(H_{1}\right), \beta\left(H_{2}\right)\right)<\min \left(\beta\left(H_{1}-N[u]\right), \beta\left(H_{2}-N[v]\right)\right)=\beta(G-N[u]-N[v])$.

This is again contradiction. Hence we have proved that $\beta(H)>\beta(G)$.

The second claim follows from the simple identity [9]

$$
-I^{\prime}(G, z)=\sum_{u \in V(G)} I(G-N[u], z) .
$$

Since all $I(G-N[u], z)$ are positive in the interval $[0, \beta(G)]$, we have

$$
I^{\prime}(G, \beta(G))<0,
$$

thus $\beta(G)$ is a simple root of $I(G, z)$.

Now we are ready to prove Theorem 1.1.

Proof of Theorem 1.1. Clearly, it is enough to prove the claim for a connected graph $G$ since the roots of $I(G, z)$ are the union of the roots $I\left(H_{j}, z\right)$ where $H_{j}$ are the connected components of the graph $G$. We can also assume that $|V(G)| \geq 2$, since the claim is trivial for $K_{1}$.

Let $u$ be an arbitrary vertex of the graph $G$. Note that $G-N[u]$ is a proper induced subgraph of $G-u$ since $G$ is a connected graph on at least two vertices.

Once again we use the identity

$$
g(z)=\frac{I(G-u, z)}{I(G, z)}=\frac{1}{1-z \frac{I(G-N[u], z)}{I(G-u, z)}} .
$$

Let us examine

$$
f(z)=z \frac{I(G-N[u], z)}{I(G-u, z)} .
$$

Since $G-N[u]$ is a proper induced subgraph of $G-u$, the convergence radius of $f(z)$ is $\beta(G-u)$, and all the coefficients of $f(z)$ are positive except the coefficient of $z^{0}$. If $\rho$ is a root of $I(G, z)$ of modulus $\beta(G)$, then it must be a singularity of $g(z)$ since $I(G-u, z)$ has no root of smaller modulus than $\beta(G-u)$, and $\beta(G-u)>\beta(G)$. Hence $f(\rho)=1$. Hence $|f(\rho)|=f(|\rho|)$ so we can use the Lemma 2.3 to obtain that $\rho=\beta(G) e^{2 \pi i r / p}$, where $p$ is some period of $f(z)$. But $f(z)$ is aperiodic: all the coefficients are positive. Thus $p=1$ and $\rho=\beta(G)$. 


\section{Concluding Remarks}

In this last section we mention that we could have proved the same results for the following weighted version of the independence polynomial.

Let $w: V(G) \rightarrow \mathbb{R}^{+}$be a positive function and let

$$
I((G, w) ; t)=\sum_{I \in \mathcal{I}}\left(\prod_{u \in I} w(u)\right)(-t)^{|I|}
$$

the weighted independence polynomial of the graph $G$, where the summation goes over all independent sets of the graph $G$ including the empty set.

The weighted independence polynomial satisfies the recursions

$$
I((G, w) ; t)=I((G-v, w) ; t)-w_{v} t I((G-N[v], w) ; t)
$$

and

$$
I((G, w) ; t)=I((G-e, w) ; t)-w_{u} w_{v} t^{2} I((G-N[u]-N[v], w) ; t),
$$

where $v$ is a vertex and $e=(u, v)$ is an arbitrary edge. From these formulas one can see that the proof of Lemma 3.2 easily generalizes and one can prove that the weighted independence polynomial has a unique root of smallest modulus, which is real.

The importance of this weighted independence polynomial (or independenceset polynomial) is that it is related to the Lovász local lemma through the following theorem of Scott and Sokal [13, 14].

We say that a graph $\mathrm{G}$ with vertex set $V$ is a dependency graph for the events $\left(A_{v}\right)_{v \in V}$ if, for each $v \in V$, the event $A_{v}$ is independent from the collection $\left\{A_{u} \mid u \in N[v]\right\}$. Let us say that a sequence $\mathbf{p}=\left(p_{v}\right)_{v \in V} \in[0,1]^{V}$ is good for the dependency graph $G$ if for every collection $\left(A_{v}\right)_{v \in V}$ of events with dependency graph $G$ such that $\mathbb{P}\left(A_{v}\right) \leq p_{v}$ for all $v \in V$, we have $\mathbb{P}\left(\cap_{v \in V} \overline{A_{v}}\right)>0$.

Now combining Theorem 1.3 (The equivalence theorem) with Theorem 2.2 (The fundamental theorem) of [14] we get the following result.

Theorem 4.1. Let $G$ be a finite graph with vertex set $V$, and let $\boldsymbol{p}=$ $\left(p_{v}\right)_{v \in V} \in[0,1]^{V}$. Then the following two statements are equivalent:

(a) $\boldsymbol{p}$ is good for the dependency graph $G$,

(b) $I((G, \boldsymbol{p}), t)>0$ for $t \in[0,1]$.

It is not clear that the weighted version of Theorem 1.1 has any relevance in the context of Scott-Sokal theorem.

Acknowledgment. I am very grateful to Vladimir Nikiforov for proposing me this problem and for lots of remarks and suggestions.

\section{REFERENCES}

[1] P. Cartier and D. Foata: Problèmes combinatoires de commutation et rèarrangements, Lecture notes in Mathematics 85 (1969), Springer-Verlag

[2] D. C. Fisher: The number of words of length $n$ in a free 'semi-Abelian' monoid, Amer. Math. Monthly 96 (1989), pp. 610-614 
[3] D. C. Fisher: Lower bounds on the number of triangles in a graph, Journal of Graph Theory, 13 (4) (1989), pp. 505-512

[4] D. C. Fisher and J. Ryan: Bounds on the largest root of the matching polynomial, Discrete Mathematics 110 (1992) No. 1-3, pp. 275-278

[5] D. C. Fisher and A. E. Solow: Dependence polynomials, Discrete Mathematics 82 (1990), No. 3 , pp. 251-258

[6] P. Flajolet and R. Sedgewick: Analytic Combinatorics, Cambridge University Press, 2009

[7] M. Goldwurm and M. Santini: Clique polynomials have a unique root of smallest modulus, Information Processing Letters, 75 (3) (2000), pp. 127-132

[8] H. Hajiabolhassan and M. L. Mehrabadi: On clique polynomials, Australasian J. Combin. 18 (1998), pp. 313-316

[9] V. E. Levit and E. Mandrescu: The independence polynomial of a graph- a survey In: Proceedings of the 1st International Conference on Algebraic Informatics. Held in Thessaloniki, October 20-23, 2005 (ED. S. Bozapalidis, A. Kalampakas and G. Rahonis), Thessaloniki, Greece: Aristotle Univ. pp. 233-254, 2005

[10] V. Nikiforov: The number of cliques in graphs of given order and size, Trans. Amer. Math. Soc. 363 (3) (2011), pp. 1599-1618

[11] A. Razborov: On the minimal density of triangles in graphs, Combin. Probab. Comput. 17 (4) (2008), pp. 603-618

[12] C. Reiher: The clique density theorem, preprint

[13] A. D. Scott and A. D. Sokal: The repulsive lattice gas, the independent-set polynomial, and the Lovász local lemma. J. Stat. Phys. 118 (2005), No. 5-6, pp. 1151-1261

[14] A. D. Scott and A. D. Sokal: On dependency graphs and the lattice gas, Combin. Probab. Comput. 15 (2006), pp. 253-279

Eötvös Loránd University, Department of Computer Science, H-1117 Budapest, Pázmány Péter sétány 1/C, Hungary \& Alfréd Rényi Institute of Mathematics, H-1053 Budapest, Reáltanoda u. 13-15, Hungary

E-mail address: csiki@cs.elte.hu 